\title{
A two-species predator-prey model in an environment enriched by a biotic resource
}

\author{
$\begin{array}{lll}\text { H. M. Safuan } & \text { H. S. Sidhu } & \text { Z. Jovanoski } \\ & & \end{array}$ \\ I. N. Towers ${ }^{4}$
}

(Received 9 November 2012; revised 20 June 2014)

\begin{abstract}
Classical population growth models assume that the environmental carrying capacity is a fixed parameter, which is not often realistic. We propose a modified predator-prey model where the carrying capacity of the environment is dependent on the availability of a biotic resource. In this model both populations are able to consume the resource, thus altering the environment. Stability, bifurcation and numerical analyses are presented to illustrate the system's dynamical behaviour. Bistability occurs in certain parameter regions. This could describe the transition from a beneficial environment to a detrimental one. We examine special cases of the system and show that both permanence and extinction are possible.
\end{abstract}

http://journal . austms.org.au/ojs/index.php/ANZIAMJ/article/view/6376 gives this article, (c) Austral. Mathematical Soc. 2014. Published August 5, 2014, as part of the Proceedings of the 16th Biennial Computational Techniques and Applications Conference. ISSN 1446-8735. (Print two pages per sheet of paper.) Copies of this article must not be made otherwise available on the internet; instead link directly to this URL for this article. 


\section{Contents}

1 Introduction

C769

2 Two-species model

C771

3 Equilibrium and stability

C772

4 Singularity theory

C774

5 Results

C776

6 Conclusion

C782

A Appendix

C783

References

C784

\section{Introduction}

The concept of the carrying capacity is extensively used in population ecology. It represents the maximum theoretical population that is sustained by an environment. Within the classical logistic model the carrying capacity is a constant, which is not often realistic since environmental conditions fluctuate over time (e.g., seasonal changes) [1]. Time dependent carrying capacities were successfully used in a number of applications $[2,3,4,5]$.

Other studies considered the carrying capacity as a state-variable. This is accomplished by introducing an additional differential equation for the rate of change of the carrying capacity. For example, Huzimura and Matsuyama [6] modelled the rate of change of the carrying capacity that decays proportionally to the population size. Thornley and France [7] and Thornley et al. [8] modelled the rate of change of the carrying capacity that decays proportionally to 
the difference between the population and its instantaneous carrying capacity. Safuan et al. [9] modelled the inter-dependency between the population and its carrying capacity by means of the coupled differential equations

$$
\begin{aligned}
& \frac{d X}{d t}=r X\left(1-\frac{X}{K}\right), \\
& \frac{d K}{d t}=a K-b K X .
\end{aligned}
$$

The first equation describes the logistic growth of population $X$ with a growth rate $r$ and is limited by a biotic resource $K$. The second equation describes the rate of change of this biotic resource. The constant $\mathbf{a}$ in equation (1b) is the self-replicating rate of the resource and the rate of uptake of the resource $\mathrm{K}$ by population $X$ is denoted by the constant $b$. In this model, the population modifies its environment to it own detriment. This model belongs to the class of so called ratio-dependent models $[10,11]$.

We extend our single-species population model [9] to a two-species model in an environment, characterised by its carrying capacity, that is dependent on the availability of a biotic resource. We seek to understand how inter-species competition affects the environment and vice versa. It is crucial to identify any environmental threshold effects which may drive one or both species to extinction. 


\section{Two-species model}

The proposed model is an extension of the single species model of Safuan et al. [9] and Basener and Ross [11],

$$
\begin{aligned}
& \frac{d X}{d t}=r_{1} X\left(1-\frac{X}{p K}\right)-a X Y, \\
& \frac{d Y}{d t}=r_{2} Y\left(1-\frac{Y}{q K}\right)+b X Y, \\
& \frac{d K}{d t}=c K-d K^{2}-e K X-f K Y .
\end{aligned}
$$

The two populations, $X$ (prey) and $Y$ (predator), grow logistically with growth rates $r_{1}$ and $r_{2}$, respectively, and their growth is limited by the availability of a biotic resource $\mathrm{K}$. The constant $\mathrm{a}$ is the maximal predator per capita consumption rate and $b$ is the rate of uptake of $X$ by $Y$. The terms $p K$ and $q \mathrm{~K}$ set the environmental carrying capacity for $\mathrm{X}$ and $\mathrm{Y}$, respectively. Here, $0<p<1$ and $0<q<1$ with $p+q=1$ so that $p K+q K=K$ is the total carrying capacity. If $p>q$, then species $X$ has access to a higher proportion of the biotic resource and can bear to be more crowded than species $Y$. Equal carrying capacity $(p=q=1 / 2)$ was discussed by Lacitignola and Tebaldi [12]. In equation (2c), the biotic resource grows logistically with developmental rate $\mathrm{c}$ and carrying capacity $\mathrm{c} / \mathrm{d}$, the biotic resource is also consumed by both $X$ and $Y$ with the uptake constants $e$ and $f$, respectively. This scenario may be thought of as a three-species food chain model [13]. All parameters are greater than or equal to zero.

Since we may think of $K$ as the instantaneous carrying capacity of a replenishing environment that is also being depleted by the presence of $X$ and $Y$, increases or decreases in $\mathrm{K}$ could affect one or both of $\mathrm{X}$ and $\mathrm{Y}$. Our aim is to understand the dynamical behaviour that (2) is capable of displaying.

On introducing nondimensional variables

$$
x=\frac{b X}{r_{1}}, \quad y=\frac{a Y}{r_{1}}, \quad k=\frac{b p K}{r_{1}}, \quad \tau=r_{1} t,
$$


model (2) becomes

$$
\begin{aligned}
& x^{\prime}=x\left(1-\frac{x}{k}\right)-x y, \\
& y^{\prime}=\alpha y\left(1-\frac{\beta y}{k}\right)+x y, \\
& k^{\prime}=\gamma k-\delta k^{2}-\epsilon k x-\phi k y,
\end{aligned}
$$

where the prime indicates a temporal derivative and

$$
\alpha=\frac{r_{2}}{r_{1}}, \quad \beta=\frac{b p}{a q}, \quad \gamma=\frac{c}{r_{1}}, \quad \delta=\frac{d}{b p}, \quad \epsilon=\frac{e}{b}, \quad \phi=\frac{f}{a} .
$$

In system (3): $\alpha$ is the ratio of growth rates of $y$ to $x ; \beta$ is the ratio of feeding rate of $y$ to the death rate of $x$ in proportion to their carrying capacities; $\gamma$ is the ratio of developmental rate of the carrying capacity to the growth rate of $x ; \delta$ is the ratio of the saturation rate of the carrying capacity to the feeding rate of $y$ in proportion to their carrying capacities; $\epsilon$ measures the relative strength of the consumption rate of the resource by $x$ to the feeding rate of $y$ on $x$; and $\phi$ is the ratio of the consumption rate of the resource by $y$ to the removal rate of $x$ due to $y$.

\section{$3 \quad$ Equilibrium and stability}

The system of ordinary differential equations (ODEs) (3) has either four or six equilibria in the $(x, y, k)$ phase-space. These equilibria lie on the steady-state solution branches

$$
\begin{array}{ll}
\mathrm{P}_{1}=\left(0,0, \frac{\gamma}{\delta}\right), & \mathrm{P}_{2}=\left(\frac{\gamma}{\delta+\epsilon}, 0, \frac{\gamma}{\delta+\epsilon}\right), \\
\mathrm{P}_{3}=\left(0, \frac{\gamma}{\beta \delta+\phi}, \frac{\beta \gamma}{\beta \delta+\phi}\right), & \mathrm{P}_{4}=\left(\frac{\alpha \hat{\mathrm{k}}(\beta-\hat{\mathrm{k}})}{\alpha \beta+\hat{k}^{2}}, \frac{\hat{k}(\alpha+\hat{k})}{\alpha \beta+\hat{k}^{2}}, \hat{k}\right),
\end{array}
$$


where $\hat{k}$ is a root of the cubic polynomial

$$
\delta \hat{k}^{3}+(\phi-\gamma-\alpha \epsilon) \hat{k}^{2}+\alpha(\beta \epsilon+\beta \delta+\phi) \hat{k}-\alpha \beta \gamma=0 .
$$

As the parameter values are varied, there exist one equilibrium point for each of $P_{1}, P_{2}$ and $P_{3}$ whereas there can be either one or three equilibria for $P_{4}$ based on the cubic equation (5).

Ecologically, the equilibrium point $P_{1}$ represents a steady-state that is free from both predator and prey, $\mathrm{P}_{2}$ is free from predator only, while $\mathrm{P}_{3}$ is free from prey only. The coexistence of predator and prey is represented by the steady-state solution branch $P_{4}$. The latter is physically realistic $(x>0)$ when $\beta>\hat{k}$, which holds for some finite interval over $\gamma$.

The stability is determined from the eigenvalues of the Jacobian matrix at an equilibrium solution $\left(x^{*}, y^{*}, k^{*}\right)$,

$$
J\left(x^{*}, y^{*}, k^{*}\right)=\left[\begin{array}{ccc}
1-\frac{2 x^{*}}{k^{*}}-y^{*} & -x^{*} & \frac{x^{* 2}}{k^{* 2}} \\
y^{*} & \alpha-\frac{2 \alpha \beta y^{*}}{k^{*}}+x^{*} & \frac{\alpha \beta y^{* 2}}{k^{* 2}} \\
-\epsilon k^{*} & -\phi k^{*} & \gamma-2 \delta k^{*}-\epsilon x^{*}-\phi y^{*}
\end{array}\right] .
$$

The eigenvalues for $P_{1}$ and $P_{2}$ are

$$
\begin{aligned}
& E_{1}=\{\alpha,-\gamma, 1\} \\
& E_{2}=\left\{\alpha+\frac{\gamma}{\delta+\epsilon},-\frac{1}{2}\left(\frac{\gamma \delta}{\delta+\epsilon}+1\right) \pm \frac{1}{2} \sqrt{\left(\frac{\gamma \delta}{\delta+\epsilon}+1\right)^{2}-4 \gamma}\right\},
\end{aligned}
$$

respectively. Then $P_{1}$ and $P_{2}$ are unstable as at least one of the eigenvalues is positive, and for this reason will no longer be considered in our analysis.

For $\mathrm{P}_{3}$ the eigenvalues are

$$
E_{3}=\left\{1-\frac{\gamma}{\beta \delta+\phi},-\frac{1}{2}\left(\frac{\beta \gamma \delta}{\beta \delta+\phi}+\alpha\right) \pm \frac{1}{2} \sqrt{\left(\frac{\beta \gamma \delta}{\beta \delta+\phi}+\alpha\right)^{2}-4 \alpha \gamma}\right\}
$$


From the structure of the eigenvalues of $P_{3}$ the condition $\gamma>\beta \delta+\phi$ ensures that it is stable, and unstable otherwise. Further, $P_{3}$ is a stable spiral if the expression under the square root sign is negative, and a stable node otherwise.

For the equilibrium points on the solution branch $\mathrm{P}_{4}$, general expressions for the eigenvalues could not be obtained explicitly. However, Appendix A discusses the special cases used in this article. Further, the condition for stability of $\mathrm{P}_{4}$ is complicated as it exhibits hysteresis which is discussed in more detail in the next section. Nevertheless, a necessary (but not sufficient) condition for stability is $\gamma<\beta \delta+\phi$.

In our investigation, $\gamma$ is the primary bifurcation parameter and all other parameters are candidates as secondary bifurcation parameters. Using $\gamma$ as bifurcation parameter for (3) results in changing the ratio of $c$ and $r_{1}$ in the original model (2). However, changing $r_{1}$ also results in changing $\alpha$ in model (3). The actual bifurcation parameter is the developmental rate $c$ of the unscaled model (2), which results in changing $\gamma$.

By adjusting the developmental rate of the carrying capacity, we manipulate the size of $x$ and $y$. If population $x$, for example, is considered as being damaging to the environment, then it is important to establish a threshold level for $\gamma$ beyond which the deleterious effects of $x$ on the environment are reduced or eliminated.

\section{Singularity theory}

Singularity theory is applied to find regions which show different steady-state behaviour. A steady-state solution of the system (3) written in the form of a reduced singularity function [14] is

$$
\mathrm{G}(\mathrm{k}, \gamma, \mathbf{p})=0,
$$

where $\mathrm{k}$ is the state-variable, $\gamma$ is the primary bifurcation parameter and $\mathbf{p}$ represents all other parameters (the secondary bifurcation parameters). The 
graph of $k$ against $\gamma$ for fixed $\mathbf{p}$ is called a steady-state diagram. The objective in studying (6) is to identify the different types of steady-state behaviour and their location in the bifurcation diagram. There are several important singularities, these include the cusp and isola [14]. In all cases, the primary singularity conditions $G=G_{k}=0$ are assumed to be satisfied. Additional conditions are invoked to distinguish between the different types of singularities.

For the equilibrium point $P_{3}$ no singularity function $G(k, \gamma, p)$ exists for which the primary singularity conditions are satisfied. On the other hand, for $\mathrm{P}_{4}$, using the cubic equation (5) we write the singularity function as

$$
\mathrm{G}=\delta \mathrm{k}^{3}+(\phi-\gamma-\alpha \epsilon) \mathrm{k}^{2}+\alpha(\beta \epsilon+\beta \delta+\phi) \mathrm{k}-\alpha \beta \gamma=0 .
$$

It is now quite easy to show, for example, that the isola does not exist: $\mathrm{G}_{\gamma}=-\left(\mathrm{k}^{2}+\alpha \beta\right) \neq 0$ [14]. We focus on the existence of the cusp singularity as this type of singularity may indicate the existence of bistability (hysteresis phenomenon).

The cusp singularity is found when G satisfies the additional conditions [14]

$$
\mathrm{G}_{\mathrm{kk}}=0 \text { with } \mathrm{G}_{\gamma} \neq 0 \text { and } \mathrm{G}_{\mathrm{kkk}} \neq 0 .
$$

When a cusp curve is crossed, the number of limit points (or turning points) on the steady-state curve changes, that is, limit points appear and disappear when moving from one region to the next. Combining the primary singularity conditions with those of (8) leads to

$$
\begin{aligned}
\mathrm{G} & =\delta \mathrm{k}^{3}+(\phi-\gamma-\alpha \epsilon) \mathrm{k}^{2}+\alpha(\beta \epsilon+\beta \delta+\phi) \mathrm{k}-\alpha \beta \gamma=0, \\
\mathrm{G}_{\mathrm{k}} & =3 \delta \mathrm{k}^{2}+2(\phi-\gamma-\alpha \epsilon) \mathrm{k}+\alpha(\beta \epsilon+\beta \delta+\phi)=0, \\
\mathrm{G}_{\mathrm{kk}} & =6 \delta \mathrm{k}+2(\phi-\gamma-\alpha \epsilon)=0
\end{aligned}
$$

where $\mathrm{G}_{\gamma}=-\left(\mathrm{k}^{2}+\alpha \beta\right) \neq 0$, and $\mathrm{G}_{\mathrm{kkk}}=6 \delta \neq 0$.

Until now our analysis has been general. To make further progress we make the simplification, $\phi=0$. The ecological consequence is that the resource is 
not consumed by the predator (see equation (3c)). Solving equations (9), we find

$$
\begin{aligned}
& \gamma=\frac{\beta(8 \delta-\epsilon)(\delta+\epsilon)^{2}}{27 \delta \epsilon}, \\
& k=\frac{\beta(8 \delta-\epsilon)(\delta+\epsilon)}{9 \delta \epsilon}, \\
& \alpha=\frac{\beta(8 \delta-\epsilon)^{2}(\delta+\epsilon)}{27 \delta \epsilon^{2}} .
\end{aligned}
$$

We require $\epsilon<8 \delta$ to ensure that the state-variable $k$ and the parameters $\gamma$ and $\alpha$ be nonnegative.

As system (3) has a large number of parameters we reduce the dimensionality of $\mathbf{p}$ by exploring a two-parameter bifurcation diagram by fixing $\phi=0$ and $\beta=\delta=1$.

Figure 1 shows the $(\epsilon, \alpha)$-parameter plane obtained from equation (10c). The curve denoting the cusp locus divides the parameter space into two regions. Region $\mathrm{A}$ is where the steady-state curve corresponding to $\mathrm{P}_{4}$ does not possess limit points, and region $\mathrm{B}$ is where limit points exist.

If $\phi>0$, then $y$ also consumes the biotic resource, and so it too degrades the environment. The dashed line in Figure 1, obtained using the software package XPP-Aut [15], represents the cusp locus for $\phi=2$. Noticeably, region A becomes larger as $\phi$ increases. The case for $\phi>0$ will no longer be discussed.

\section{Results}

First we choose a case in region A when $\epsilon=3$ and $\alpha=0.1$. The software XPPAut was used to generate two steady-state branches denoted by $\mathrm{P}_{3}$ (red) and $\mathrm{P}_{4}$ (blue), as shown in Figure 2. A transcritical bifurcation (ТВ) occurs at $\gamma_{\mathrm{тв}}=1$. 


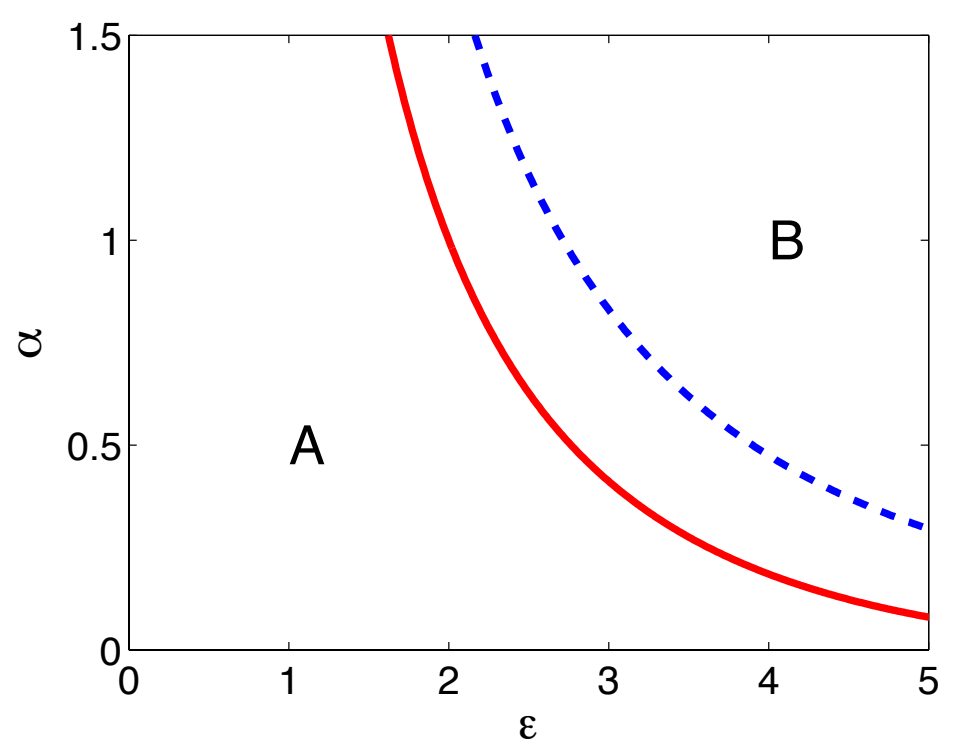

Figure 1: Two-parameter bifurcation diagram when $\phi=0$ (red solid line) and $\phi=2$ (blue dashed line) with $\beta=\delta=1$; region $\mathrm{A}$ - no bistability; region B-bistability exists.

As $\gamma$ increases in magnitude, the branch $\mathrm{P}_{4}$ is stable below the threshold value, $\gamma<\gamma_{\text {тв }}$, whereas branch $\mathrm{P}_{3}$ becomes stable for $\gamma>\gamma_{\text {тв }}$.

For increasing values of $\gamma$, the equilibrium value of the carrying capacity $k$ also increases, as shown in Figure 2(a). The values of $y$ and $x$ also increase since the environment becomes more conducive to growth, as represented by the curves $\mathrm{P}_{4}$ in Figure 2(b) and 2(c). As $\gamma$ continues to increase, so do $\mathrm{k}$ and $\boldsymbol{y}$, and the equilibrium population size of $x$ reaches a maximum before declining due to predation by $y$. Passing through the threshold, $\gamma_{\text {тв }}, k$ and $y$ continue to increase following branch $\mathrm{P}_{3}$. However, population $x$ dies out leaving only the dominant population $y$ to survive in the rich biotic environment. These results show that the survival of population $x$ is determined by the bifurcation parameter $\gamma$. If $x$ is considered as a harmful (or not desirable) population, 
then a rich environment will guarantee its elimination.

To illustrate the behaviour in region $\mathrm{B}$, we keep $\epsilon=3$ and increase $\alpha$ from 0.1 to 1.5. Physically, increasing $\alpha$ has the effect of increasing the growth rate of $y$ relative to $x$. The steady-state curves are shown in Figure 3. Moving from region A to B, two limit points (LP1 and LP2) exist on the S-shaped steady-state curve $\mathrm{P}_{4}$. Only the first limit point (LP1) is physically meaningful since the second limit point (LP2) occurs for $x<0$ (see Figure 3(c)). These latter solutions are unstable and will not be discussed further.

Within region B, bistability can occur for a certain range of $\gamma$. From Figure 3, bistability occurs for $1<\gamma<1.184$. In other words, for values of $\gamma$ between the transcritical bifurcation (TB) and the limit point LP1, depending on the choice of initial conditions, the solution can approach the stable equilibria on the steady-state solution branch $\mathrm{P}_{3}$ or $\mathrm{P}_{4}$. This bistable behaviour could determine the transition between a favourable and an unfavourable environment for the two populations.

To illustrate bistability we choose two different initial conditions for the prey in the vicinity of LP1. Figure 4 shows the time series solutions of system (3) with parameters used in Figure 3 at the specific value of $\gamma=1.1$. For the initial condition $\left(x_{0}, y_{0}, k_{0}\right)=(0.01,0.1,0.2)$, both $k$ and $y$ increase until reaching their steady-state values, whereas, the prey population $x$ asymptotically decays to zero. However, for a slight change in the initial condition $\left(x_{0}, y_{0}, k_{0}\right)=(0.02,0.1,0.2)$ the result is quite different. The prey $x$ also increases until reaching its steady-state value.

Bistability was previously reported in bacterial populations. Malka et al. [16] modelled the dynamics of bacterium and phagocyte populations and found bistability regions that indicate a healthy or an infected state of a host. Elf et al. [17] studied the response of bacteria towards antibiotics and found bistable behaviour in the bacterial growth rate. Studies by Dubnau and Losick [18] and Santillán [19] also discussed bistability in their bacterial growth studies. 


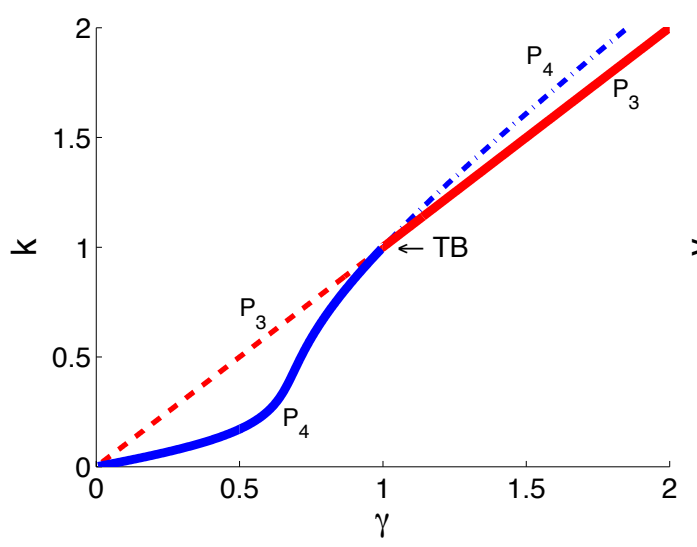

(a)

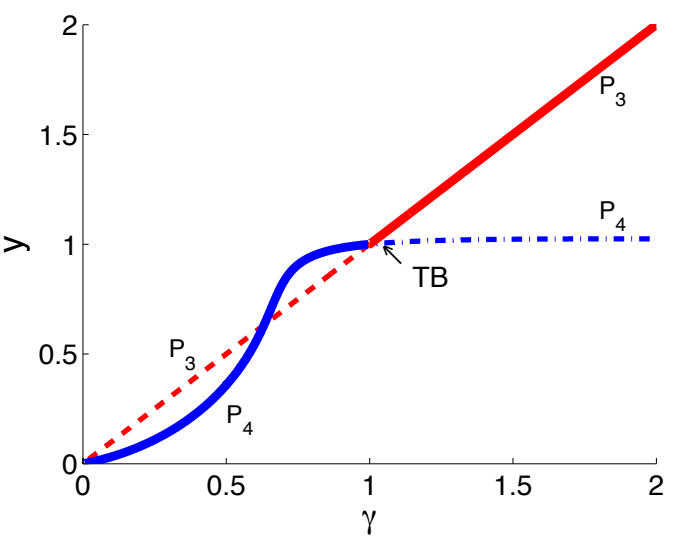

(b)

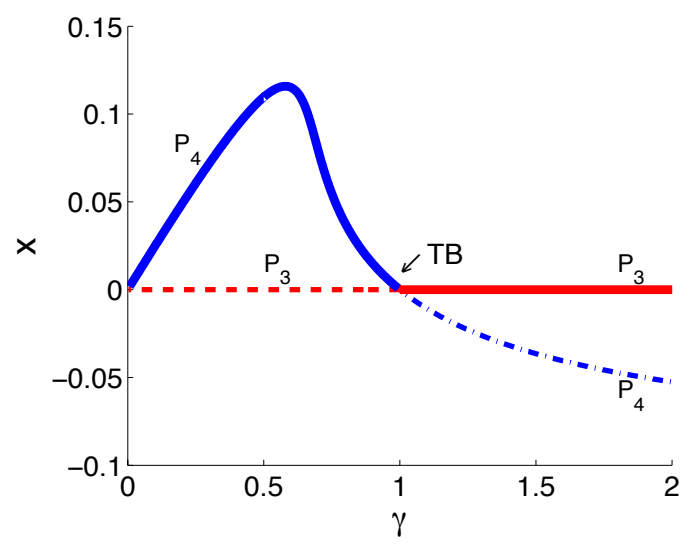

(c)

Figure 2: Steady-state diagrams for (a) k; (b) $y$; and (c) $x$; and with $\phi=0$, $\beta=\delta=1, \epsilon=3$ and $\alpha=0.1$. Solid lines are stable steady-state solutions, dashed lines are unstable and dashed-dot lines represent unphysical solutions. 


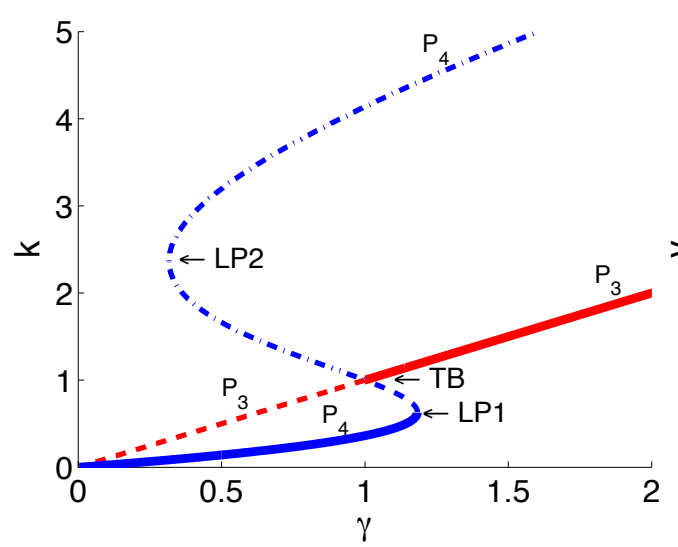

(a)

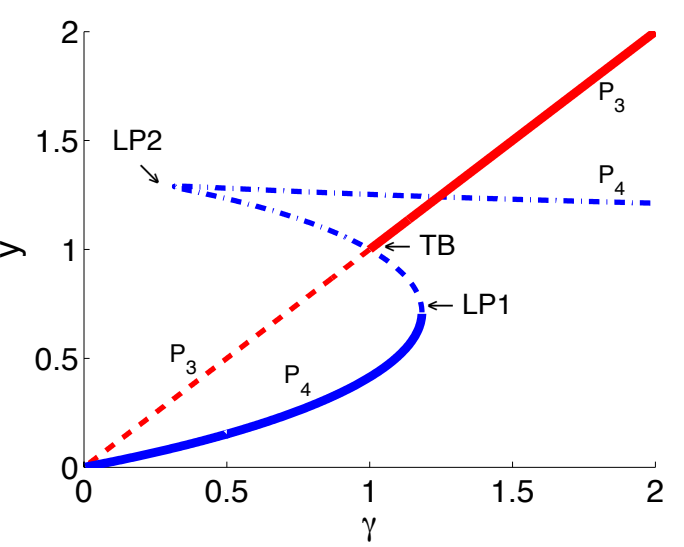

(b)

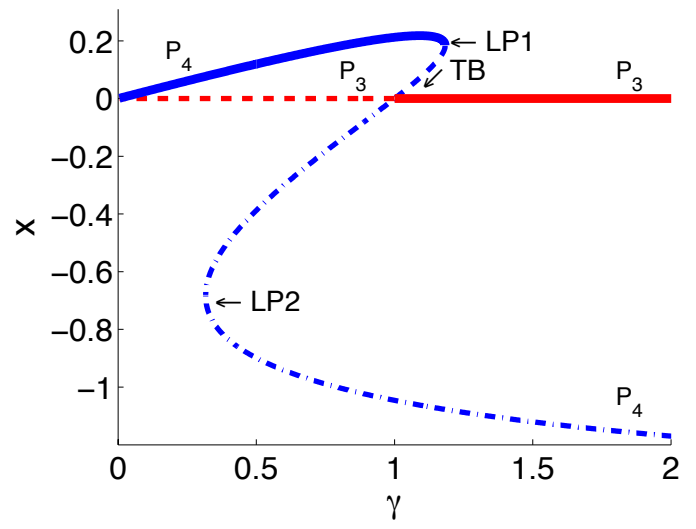

(c)

Figure 3: Steady-state diagrams for (a) k; (b) $y$; and (c) $x$; and with parameters like Figure 2, $\phi=0, \beta=\delta=1, \epsilon=3$, except $\alpha=1.5$. Solid lines are stable steady-state solutions, dashed lines are unstable and dashed-dot lines represent unphysical solutions. 


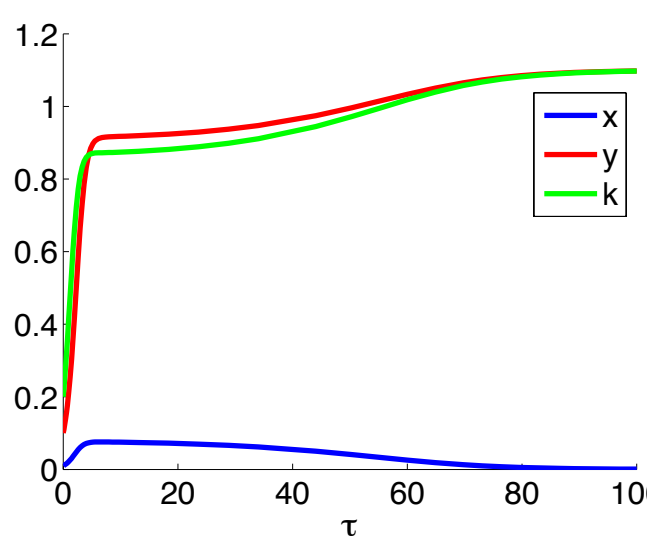

(a)

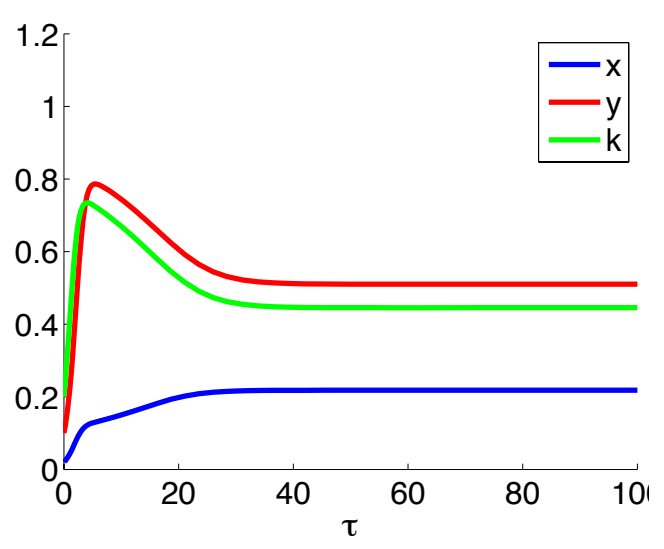

(b)

Figure 4: Time plots of two initial conditions $\left(x_{0}, y_{0}, k_{0}\right)$ in the bistablity region, $\gamma=1.1$ for (a) $(0.01,0.1,0.2)$ and $(b)(0.02,0.1,0.2)$.

At this stage it is worth contrasting model (3) with that of a model with constant carrying capacity, $k$. The equilibrium points of a model with constant carrying capacity are

$$
\begin{array}{ll}
P_{1}^{\prime}=(0,0), & P_{2}^{\prime}=(k, 0), \\
P_{3}^{\prime}=\left(0, \frac{k}{\beta}\right), & P_{4}^{\prime}=\left(\frac{\alpha k(\beta-k)}{k^{2}+\alpha \beta}, \frac{(\alpha+k) k}{k^{2}+\alpha \beta}\right) .
\end{array}
$$

Here, $P_{1}^{\prime}$ and $P_{2}^{\prime}$ are unstable. If $k>\beta$, then $P_{3}^{\prime}$ is stable and $P_{4}^{\prime}$ is unstable. For $k<\beta, P_{3}^{\prime}$ is unstable and $P_{4}^{\prime}$ is stable. There exist a transcritical bifurcation when $k=\beta$. This means that for $k<\beta$ the predator and prey co-exist; however, for a sufficiently large carrying capacity $(k>\beta)$ the prey dies out. This scenario is qualitatively similar to the results of region A of model (3). Importantly, a model with constant $k$ does not exhibit bistability. 


\section{Conclusion}

This article investigates a two-species population model with competition in an environment enriched by a biotic resource. The biotic resource affects the carrying capacity of each species; however, the species directly alter the amount of resource available by interacting with it. Stability analysis with the aid of singularity theory provides the tools in understanding the effects of the environmental carrying capacity on the two populations. The environmental development rate, $\gamma$, is a critical parameter in the system (3). It determines whether or not population $x$ say, becomes extinct.

Bistability is found to exist for a certain region of parameters. To maintain both populations in the system, $\gamma$ has to be less than the threshold value at the transcritical bifurcation point, TB. If the effect of population $y$ is neutral $(\phi=0)$ on the environment, then increasing the developmental rate $(\gamma)$ will guarantee its survival at the expense of the harmful population $x$.

This feature of our model, that the prey population can become extinct when the enrichment parameter exceeds some threshold value, is reminiscent of the "paradox of enrichment" first observed by Rosenzweig [20]. The paradox roughly says that in a predator-prey system, increasing the nutrition to the prey may lead to an extinction of both the prey and the predator. The extinction of both the prey and predator, due to an oscillatory instability following an enrichment in the carrying capacity, does not arise in our model. A change of stability occurs but the predator population remains stable.

Acknowledgements: HMS is grateful to Universiti Tun Hussein Onn Malaysia, the School of Physical, Environmental and Mathematical Sciences, UNSW Canberra and the ANZIAM/CSIRO Student Support Scheme for financial support and Thiansiri Luangwilai for his helpful discussion on the bifurcation and numerical analyses. 


\section{A Appendix}

The non-trivial steady-state solution branch $\mathrm{P}_{4}$ is

$$
(\hat{x}, \hat{y}, \hat{k})=\left(\frac{\alpha \hat{k}(\beta-\hat{k})}{\alpha \beta+\hat{k}^{2}}, \frac{\hat{k}(\alpha+\hat{k})}{\alpha \beta+\hat{k}^{2}}, \hat{k}\right),
$$

where $\hat{k}$ is a root of the cubic polynomial

$$
\delta \hat{k}^{3}+(\phi-\gamma-\alpha \epsilon) \hat{k}^{2}+\alpha(\beta \epsilon+\beta \delta+\phi) \hat{k}-\alpha \beta \gamma=0 .
$$

In this appendix, we perform numerical calculations to determine the eigenvalues of the corresponding equilibria that lie on the $\mathrm{P}_{3}$ and $\mathrm{P}_{4}$ steady-state solution branches using the parameter set used in the text $(\alpha=1.5, \beta=\delta=1$, $\epsilon=3, \phi=0)$.

According to Figure 3(a), with $\gamma$ in the bistability region, $1<\gamma<1.184$, the cubic polynomial (11) has three positive roots, all residing on a different branch of $P_{4}$. If we set $\gamma=1.1$, then we have $P_{3}=(0,1.1,1.1)$. The approximate roots of (11) are $\hat{k}_{1}=0.45, \hat{k}_{2}=0.86$, and $\hat{k}_{3}=4.29$, with the corresponding points on $\mathrm{P}_{4}$ at $\mathrm{P}_{4_{1}}=(0.22,0.51,0.45), \mathrm{P}_{4_{2}}=(0.08,0.91,0.86)$, and $P_{4_{3}}=(-1.06,1.25,4.29)$, respectively.

There are six equilibria: $P_{1}, P_{2}, P_{3}$ and three lying on the $P_{4}$ solution curve, $P_{4_{1}}, P_{4_{2}}$ and $P_{4_{3}}$. As discussed in the text, $P_{1}$ and $P_{2}$ are unstable. Table 1 shows the eigenvalues of the Jacobian matrix $J$ for $P_{3}, P_{4_{1}}, P_{4_{2}}$ and $P_{4_{3}}$. Based on Table 1 , we know that $P_{3}$ is a stable node, $P_{4_{1}}$ is a stable spiral with negative real eigenvalues, whereas both $P_{4_{2}}$ and $P_{4_{3}}$ are unstable. Thus, for these parameter values bistability can only occur between steady-states $P_{3}$ and $P_{4_{1}}$, depending on the choice of initial conditions. 
Table 1: Eigenvalues of Jacobian matrices for steady-states $P_{3}$ and $P_{4}$.

\begin{tabular}{llll}
$\mathrm{P}_{3}$ & $\mathrm{P}_{4_{1}}$ & $\mathrm{P}_{4_{2}}$ & $\mathrm{P}_{4_{3}}$ \\
\hline$\lambda_{1}=-0.10$ & $\lambda_{1}=-0.24$ & $\lambda_{1}=0.06$ & $\lambda_{1}=0.86$ \\
$\lambda_{2}=-0.86$ & $\lambda_{2,3}=-1.21 \pm 0.47 i$ & $\lambda_{2,3}=-1.30 \pm 0.40 i$ & $\lambda_{2}=-1.13$ \\
$\lambda_{3}=-1.74$ & & & $\lambda_{3}=-4.21$
\end{tabular}

\section{References}

[1] J. Vandermeer. Seasonal isochronic forcing of Lotka Volterra equations. Prog. Theor. Phys., 96:13-28, 1996. doi:10.1143/PTP.96.13 C769

[2] S. Ikeda and T. Yokoi. Fish population dynamics under nutrient enrichment-A case of the East Seto Inland Sea. Ecol. Model., 10:141-165, 1980. doi:10.1016/0304-3800(80)90057-5 C769

[3] S. P. Rogovchenko and Y. V. Rogovchenko. Effect of periodic environmental fluctuations on the Pearl-Verhulst model. Chaos, Solitons, Fractals, 39:1169-1181, 2009. doi:10.1016/j.chaos.2007.11.002 C769

[4] H. Safuan, I. N. Towers, Z. Jovanoski and H. S. Sidhu. A simple model for the total microbial biomass under occlusion of healthy human skin. In Chan, F., Marinova, D. and Anderssen, R.S. (eds) MODSIM2011, 19th International Congress on Modelling and Simulation. Modelling and Simulation Society of Australia and New Zealand., 733-739, 2011. http://www.mssanz.org.au/modsim2011/AA/safuan.pdf C769

[5] P. Meyer and J. H. Ausubel. Carrying capacity: A model with logistically varying limits. Technol. Forecast. Soc., 61:209-214, 1999. doi:10.1016/S0040-1625(99)00022-0 C769

[6] R. Huzimura and T. Matsuyama. A mathematical model with a modified logistic approach for singly peaked population processes. Theor. Popul. Biol., 56:301-306, 1999. doi:10.1006/tpbi.1999.1426 C769 
[7] J. H. M. Thornley and J. France. An open-ended logistic-based growth function. Ecol. Model., 184:257-261, 2005. doi:10.1016/j.ecolmodel.2004.10.007 C769

[8] J. H. M. Thornley, J. J. Shepherd and J. France. An open-ended logistic-based growth function: Analytical solutions and the power-law logistic model. Ecol. Model., 204:531-534, 2007. doi:10.1016/j.ecolmodel.2006.12.026 C769

[9] H. M. Safuan, I. N. Towers, Z. Jovanoski and H. S. Sidhu. Coupled logistic carrying capacity model. ANZIAM J, 53:C172-C184, 2012. http://journal. austms.org.au/ojs/index.php/ANZIAMJ/article/ view/4972 C770, C771

[10] P. H. Leslie and J. C. Gower. The properties of a stochastic model for the predator-prey type of interaction between two species. Biometrika, 47:219-234, 1960. doi:10.1093/biomet/47.3-4.219 C770

[11] B. Basener and D. S. Ross. Booming and crashing populations and Easter Island. SIAM J. Appl. Math., 65:684-701, 2005. doi:10.1137/S0036139903426952 C770, C771

[12] D. Lacitignola and C. Tebaldi. Symmetry breaking effects on equilibria and time dependent regimes in adaptive Lotka-Volterra systems. Int. J. Bifurcat. Chaos, 13:375-392, 2003. doi:10.1142/S0218127403006595 C771

[13] F. Wang and G. Pang. Chaos and Hopf bifurcation of a hybrid ratio-dependent three species food chain. Chaos, Solitons, Fractals, 36:1366-1376, 2008. doi:10.1016/j.chaos.2006.09.005 C771

[14] R. Ball. Understanding critical behaviour through visualization: A walk around the pitchfork. Comput. Phys. Commun., 142:71-75, 2001. doi:10.1016/S0010-4655(01)00322-8 C774, C775

[15] B. Ermentrout. XPP-Aut v. 6.00, 2011.

http://www.math.pitt.edu/ bard/xpp/xpp.html C776 
[16] R. Malka, E. Shochat and V. R. Kedar. Bistability and bacterial infections. PLOS ONE, 5:1-10, 2010. doi:10.1371/journal.pone.0010010 C778

[17] J. Elf, K. Nilsson, T. Tenson and M. Ehrenberg. Bistable bacterial growth rate in response to antibiotics with low membrane permeability. Phys. Rev. Lett., 97:258104, 2006. doi:10.1103/PhysRevLett.97.258104 $\mathrm{C} 778$

[18] D. Dubnau and R. Losick. Bistability in bacteria. Mol. Microbiol., 61:564-572, 2006. doi:10.1111/j.1365-2958.2006.05249.x C778

[19] M. Santillán. Bistable behavior in a model of the lac Operon in Escherichia coli with variable growth rate. Biophys. J., 94:2065-2081, 2008. doi:10.1529/biophysj.107.118026 C778

[20] M. Rosenzweig. Paradox of enrichment: destabilization of exploitation ecosystem in ecological time. Science, 171:385-387, 1971. doi:10.1126/science.171.3969.385 C782

\section{Author addresses}

1. H. M. Safuan, Applied and Industrial Mathematics Research Group, School of Physical, Environmental and Mathematical Sciences, University of New South Wales, Canberra 2600, Australia. Faculty of Science, Technology, and Human Development, Universiti Tun Hussein Onn Malaysia, Johor 86400, Malaysia.

mailto:hamizahs@uthm.edu.my

2. H. S. Sidhu, Applied and Industrial Mathematics Research Group, School of Physical, Environmental and Mathematical Sciences, University of New South Wales, Canberra 2600, Australia. mailto:h.sidhu@adfa.edu.au

3. Z. Jovanoski, Applied and Industrial Mathematics Research Group, 
School of Physical, Environmental and Mathematical Sciences, University of New South Wales, Canberra 2600, Australia. mailto:z.jovanoski@adfa.edu . au

4. I. N. Towers, Applied and Industrial Mathematics Research Group, School of Physical, Environmental and Mathematical Sciences, University of New South Wales, Canberra 2600, Australia. mailto:i.towers@adfa.edu.au 